\title{
THE $\pi$-CHARACTER THEORY OF SOLVABLE GROUPS
}

\author{
I. M. ISAACS \\ (Received 28 September 1993) \\ Communicated by L. G. Kovács
}

\begin{abstract}
There is a deeper structure to the ordinary character theory of finite solvable groups than might at first be apparent. Much of this structure, which has no analog for general finite groups, becomes visible only when the characters of solvable groups are viewed from the perspective of a particular set $\pi$ of prime numbers. This purely expository paper discusses the foundations of this $\pi$-theory and a few of its applications. Included are the definitions and essential properties of Gajendragadkar's $\pi$-special characters and their connections with the irreducible $\pi$-partial characters and their associated Fong characters. Included among the consequences of the theory discussed here are applications to questions about the field generated by the values of a character, about extensions of characters of subgroups and about M-groups.
\end{abstract}

1991 Mathematics subject classification (Amer. Math. Soc.): $20 \mathrm{C} 15$.

\section{Introduction}

The purpose of this article is to discuss the growing body of results that concern the classical (complex) character theory of a solvable group $G$, as seen from the perspective of a particular set $\pi$ of prime numbers. We consider $\pi$-special characters, $\pi$-factored characters, $\pi$-partial characters and Fong characters, and we explore some of the connections among these objects. This paper is intended as a survey; it is entirely expository and contains no new theorems. We state most results without proof, but in some cases we present detailed arguments. This is done in order to give the flavor of the subject and to help clarify the interdependence of the various ideas.

In this author's opinion, it is a fair test for a mathematical theory to require that it be able to contribute to the solution of problems beyond those suggested by the theory itself. It is gratifying that the $\pi$-character theory of solvable groups passes this test, and a substantial portion of our discussion here is concerned with applications of the

(C) 1994 Australian Mathematical Society 0263-6115/94 \$A2.00+0.00 
theory.

Despite our reference to 'solvable groups' in the previous two paragraphs (and in the title of this article), we usually do not actually require our groups to be solvable. In most cases, it suffices that they be $\pi$-separable, which means that each composition factor must be either a $\pi$-group or a $\pi^{\prime}$-group. Unfortunately, there is some cost in this added generality, and only a modest benefit. We require results such as the fact that if $H$ is a Hall $\pi$-subgroup of a $\pi$-separable group $G$, then every $\pi$-subgroup of $G$ is conjugate in $G$ to a subgroup of $H$. This is true, but if we do not assume that $G$ is solvable, the only known proofs depend on the Feit-Thompson theorem that groups of odd order are solvable. Since whatever interest our theorems might have is already present when the results are stated in the solvable case, the reader is invited to ignore the generality (and the dependence on the odd-order theorem) and simply to read 'solvable' wherever we have written ' $\pi$-separable'.

\section{2. $\pi$-special characters}

Given an irreducible character $\chi$ of a group $G$, there are two positive integers naturally associated with $\chi$ that necessarily divide $|G|$. One of these, of course, is the degree $\chi(1)$ and the other is the determinantal order $o(\chi)$. (This latter number is the order of the linear character $\operatorname{det}(\chi)$ in the group of linear characters of $G$.) If we fix a set $\pi$ of primes, and we wish to consider 'special' irreducible characters of $G$ that somehow reflect the $\pi$-structure of $G$, it is natural to restrict our attention to those irreducible characters $\chi \in \operatorname{Irr}(G)$ for which both of these integers are $\pi$-numbers. That this is not a completely ridiculous thing to do is suggested by the following well known theorem of P. X. Gallagher. (See [5, Corollary 8.16].)

THEOREM 2.1. Let $N \triangleleft G$ and suppose $\theta \in \operatorname{Irr}(N)$ is invariant in $G$. Assume that $\theta(1)$ and $\mathrm{o}(\theta)$ are $\pi$-numbers and that $|G / N|$ is divisible by no prime in $\pi$. Then $\theta$ is extendible to $G$. Furthermore, there is a unique extension $\chi$ such that $\mathrm{o}(\chi)$ is $a$ $\pi$-number.

We mention that in Theorem 2.1, the extension $\chi$ of $\theta$ such that $o(\chi)$ is a $\pi$-number is called the canonical extension of $\theta$, and it is often denoted $\hat{\theta}$. Its determinantal order is actually equal to that of $\theta$.

Since we are interested in solvable groups, which are characterized by having an ample supply of normal subgroups, it is natural to consider irreducible characters which behave well with respect to the normal structure of our group $G$. We would like, therefore, to consider only normally heritable properties $\mathscr{S}$ of irreducible characters: properties that satisfy the condition: 
(NH) If $\chi \in \operatorname{Irr}(G)$ satisfies $\mathscr{P}$, and $N \triangleleft G$, then all irreducible constituents of $\chi_{N}$ satisfy $\mathscr{P}$.

Our 'special' characters which will reflect the $\pi$-structure of $G$ will be characters with $\pi$-degree, and this property is normally heritable. But condition (NH) is not automatic for the property that the determinantal order is also a $\pi$ number, and so we must impose it.

DEFINITION 2.2. Let $G$ be $\pi$-separable. Then $\chi \in \operatorname{Irr}(G)$ is $\pi$-special provided

(i) $\chi(1)$ is a $\pi$ number, and

(ii) $o(\theta)$ is a $\pi$-number for every irreducible constituent $\theta$ of $\chi_{s}$ for every subnormal subgroup $S$ of $G$.

We stress that the set $\mathscr{X}_{\pi}(G)$ of $\pi$-special characters of $G$ is defined if, but only if, $G$ is $\pi$-separable. (In particular, if $G$ is $\pi$-separable, we can speak of $\pi^{\prime}$-special characters of $G$, and if $G$ is solvable, it makes sense to consider $\pi$-special characters for arbitrary prime sets $\pi$.)

The definition of $\pi$-special characters appeared first in [6], but only in the case where $\pi=p^{\prime}$, the complement of a single prime. It was shown in that paper that $\mathscr{X}_{\pi}(G)$ is a set of canonical lifts for the $p^{\prime}$-degree irreducible $p$-Brauer characters of a $p$-solvable group $G$. This set was then used to construct a normally heritable collection of canonical lifts for all of the irreducible $p$-Brauer characters of $G$, thereby proving a strong form of the Fong-Swan theorem. (We shall have more to say about this later.)

It was D. Gajendragadkar who first considered $\pi$-special characters for arbitrary prime sets $\pi$, and he accumulated a number of striking results about these characters in his paper [4]. Probably the most surprising of Gajendragadkar's theorems is the following.

THEOREM 2.3. Let $G$ be $\pi$-separable and suppose that $\alpha$ and $\beta$ are respectively $\pi$-special and $\pi^{\prime}$-special irreducible characters of $G$. Then the product $\chi=\alpha \beta$ is irreducible. If also $\chi=\alpha^{\prime} \beta^{\prime}$ is another factorization of $\chi$ with $\alpha^{\prime}$ and $\beta^{\prime}$ respectively $\pi$-special and $\pi^{\prime}$-special, then $\alpha=\alpha^{\prime}$ and $\beta=\beta^{\prime}$.

We mention that the proof of Theorem 2.3 proceeds via induction on the order of the group. In order to prove that $\alpha \beta$ is irreducible, both parts of the inductive hypothesis must be applied for some proper normal subgroup of $G$. In other words, the uniqueness of the factorization in Theorem 2.3 is essential in proving the irreducibility of products of $\pi$-special and $\pi^{\prime}$-special characters.

Gajendragadkar's theorem suggests that it might be interesting to consider the set of $\pi$-factored irreducible characters of $G$ : those which can be written as products of $\pi$-special and $\pi^{\prime}$-special characters. We observe that the property of being $\pi$-factored is normally heritable. To see this, suppose that $N \triangleleft G$ and that $\chi=\alpha \beta$, where $\alpha$ and 
$\beta$ are respectively $\pi$-special and $\pi^{\prime}$-special characters of $G$. Then $\chi_{N}=\alpha_{N} \beta_{N}$, and it follows that an irreducible constituent $\psi$ of $\chi_{N}$ must be a constituent of $\alpha_{0} \beta_{0}$ for some irreducible constituents $\alpha_{0}$ and $\beta_{0}$ of $\alpha_{N}$ and $\beta_{N}$. But $\alpha_{0}$ and $\beta_{0}$ are respectively $\pi$-special and $\pi^{\prime}$-special, and it follows by Theorem 2.3 that $\psi=\alpha_{0} \beta_{0}$ is $\pi$-factored, as claimed.

If $G$ is the direct product of a $\pi$-group and a $\pi^{\prime}$-group, it is clear that every irreducible character of $G$ is $\pi$-factored. It is not hard to show that the converse of this statement is true too, but more interesting is the question of how common are $\pi$-factored characters in the general case of $\pi$-separable groups. (Clearly, the factorization theory would be of little use and minimal interest if $\pi$-factored characters were very rare.) Fortunately, there is a very satisfactory result in this direction.

THEOREM 2.4. Suppose $G$ is $\pi$-separable. Then every primitive irreducible character of $G$ is $\pi$-factored.

This result, which was first proved in [7], is an easy consequence (via induction) of the following general result. (This inductive argument shows that in fact, quasiprimitivity is sufficient to guarantee factorability.)

LEMMA 2.5. Let $G$ be $\pi$-separable and suppose that $N \triangleleft G$ is such that

$$
G / N=U / N \times V / N,
$$

a direct product of a $\pi$-group and a $\pi^{\prime}$-group, respectively. Suppose $\psi=\alpha \beta \in \operatorname{Ir}(N)$, where $\alpha$ is $\pi$-special and $\beta$ is $\pi^{\prime}$-special, and where $\beta$ is invariant in $U$ and $\alpha$ is invariant in $V$. Then every irreducible character $\chi \in \operatorname{Irr}(G)$ lying over $\psi$ is $\pi$-factored.

The key to the proof of Lemma 2.5 is to find conditions sufficient to guarantee that characters are $\pi$-special. The fundamental results in this direction are the following.

LEMMA 2.6. Let $G$ be $\pi$-separable, and suppose that $N \triangleleft G$ and that $\alpha \in \operatorname{Irr}(N)$ is $\pi$-special.

(a) If $G / N$ is a $\pi$-group, then every irreducible character of $G$ lying over $\alpha$ is $\pi$-special.

(b) If $G / N$ is a $\pi^{\prime}$-group and $\alpha$ is invariant in $G$, then the canonical extension $\hat{\alpha}$ of $\alpha$ to $G$ is $\pi$-special.

Lemma 2.6 can be found in this generality in [4], but we note that it appears already in [6], in the case where $\pi$ is the complement of a prime. We note that in the situation of 2.6(b), it is not hard to see that $\hat{\alpha}$ is the only $\pi$-special character of $G$ lying over $\alpha$.

Lemma 2.5 is easily proved using 2.6 , and we sketch the argument. 
PROOF OF LEMMA 2.5. Let $\hat{\beta}$ and $\hat{\alpha}$ be the canonical extensions of $\beta$ and $\alpha$ to $U$ and $V$, respectively. These extensions are $\pi^{\prime}$-special and $\pi$-special by Lemma 2.6(b), and we conclude by 2.6(a) that all irreducible constituents of $\hat{\beta}^{G}$ and $\hat{\alpha}^{G}$ are $\pi^{\prime}$-special and $\pi$-special, respectively. It follows that all irreducible constituents of $\left(\hat{\alpha}^{G}\right)\left(\hat{\beta}^{G}\right)$ are $\pi$-factored.

It suffices now to observe that $\psi^{G}=\left(\hat{\alpha}^{G}\right)\left(\hat{\beta}^{G}\right)$. This holds because

$$
(\alpha \beta)^{U}=\alpha^{U} \hat{\beta}=\left(\hat{\alpha}_{N}\right)^{U} \hat{\beta}=\left(\hat{\alpha}^{G}\right)_{N} \hat{\beta}
$$

and thus

$$
\psi^{G}=\left((\alpha \beta)^{U}\right)^{G}=\hat{\alpha}^{G} \hat{\beta}^{G}
$$

as required.

The significance of Theorem 2.4 for applications is this. Suppose that we wish to prove something about some irreducible character $\chi$ of a $\pi$-separable group $G$. It may happen that $\chi$ is induced from some character $\psi$ of a proper subgroup of $G$. In this case, and working by induction on $|G|$ or on the degree of $\chi$, we presumably have information about $\psi$ from which we can deduce the desired conclusion about $\chi$. In the remaining case, $\chi$ is primitive, and hence is $\pi$-factored, and we can write $\chi=\alpha \beta$, where $\alpha$ and $\beta$ are $\pi$-special and $\pi^{\prime}$-special, respectively. The hope here is that we know enough about special characters to deduce information about $\alpha$ and $\beta$, and thereby to prove our result about their product $\chi$.

What kind of information might we hope to know about $\pi$-special characters, beyond the mere fact that they are $\pi$-special? A useful heuristic principle is that $\pi$-special characters of $G$ 'think' that $G$ is a $\pi$-group. Not only are their degrees and determinantal orders necessarily $\pi$-numbers, but also in other respects, $\pi$-special characters behave like characters of $\pi$-groups. The key to most results of this type is the following theorem of Gajendragadkar [4].

THEOREM 2.7. Let $H$ be a Hall $\pi$-subgroup of a $\pi$-separable group $G$. Then restriction defines an injection of the set $\mathscr{X}_{\pi}(G)$ of $\pi$-special characters of $G$ into $\operatorname{IrT}(H)$.

It turns out that there is a very simple description of the image of this restriction map. We present this in Corollary 8.3.

At this point, we have discussed enough results about special characters and factorization theory to present our first (minor) application to the general character theory of solvable groups. Note that the statement of the following theorem mentions none of our technical definitions and that the result would definitely be false without the solvability assumption.

THEOREM 2.8. Let $\chi$ be a primitive character of a solvable group $G$. Then $\chi(1)^{2}$ divides $|G|$. 
PROOF. Fix a prime $p$ and let $P \in \operatorname{Syl}_{p}(G)$. It suffices to prove that $\left(\chi(1)_{p}\right)^{2}$ divides $|P|$, where we have written $\chi(1)_{p}$ to denote the $p$-part of the degree $\chi(1)$.

Since $\chi$ is primitive and $G$ is $p$-special, we can use Theorem 2.4 to write $\chi=\alpha \beta$ where $\alpha$ is $p$-special and $\beta$ is $p^{\prime}$-special. In particular, $\chi(1)=\alpha(1) \beta(1)$, and since $\alpha(1)$ is a $p$-power and $\beta(1)$ is not divisible by $p$, we see that $\chi(1)_{p}=\alpha(1)$.

Now $\alpha_{P}$ is irreducible by Theorem 2.7, and hence $|P| \geq \alpha_{P}(1)^{2}=\alpha(1)^{2}$. Since for prime powers, inequality is equivalent to divisibility, we see that $\alpha(1)^{2}$ divides $|P|$, as required.

The reader has probably noticed that the above argument actually proves more than we stated. In fact, in the situation of Theorem 2.8 , we see that $|G| / \chi(1)^{2}$ is ar integer divisible by $\prod_{p}\left|\mathbf{Z}\left(S_{p}\right)\right|$, where $S_{p}$ is some Sylow $p$-subgroup of $G$. Anothes application in somewhat the same spirit is the following result, whose proof we alsc leave to the reader.

THEOREM 2.9. Let $\chi \in \operatorname{Irr}(G)$, where $G$ is solvable, and suppose that $|G| / \chi(1)$ i: not divisible by the prime $p$. Then there exists a $p^{\prime}$-subgroup $H \subseteq G$ and a characte $\psi \in \operatorname{Irr}(H)$ such that $\chi=\psi^{G}$. In particular, $\chi$ vanishes at all elements of $G$ having order divisible by $p$.

We close this section by mentioning that for solvable groups, Gajendragadkar': irreducibility theorem (2.3) can be extended as follows.

THEOREM 2.10. Let $G$ be solvable and suppose that we have selected a p-specia character $\alpha_{p} \in \operatorname{Irr}(G)$ for each prime divisor $p$ of $|G|$. Then $\chi=\prod_{p} \alpha_{p}$ is irreducible and $\chi$ uniquely determines each of the factors $\alpha_{p}$.

This result follows directly from Theorem 2.3 using the fact that if $\alpha$ and $f$ are respectively $\pi$-special and $\sigma$-special for disjoint prime sets $\pi$ and $\sigma$, then the irreducible character $\alpha \beta$ is ( $\pi \cup \sigma$ )-special. It is not hard to see that Theorem 2.6 can also be generalized: every primitive character of a solvable group has a 'ful factorization', as in Theorem 2.10.

\section{Fields of values}

One of the most striking applications of the $\pi$-factorization theory is to the solvable case of Feit's conjecture concerning the field of values of a character. Following standard notation, we write $\mathbb{Q}(\chi)$ to denote the subfield of the complex number: obtained by adjoining to the rational field $\mathbb{Q}$ all values $\chi(g)$ of a character $\chi$ of ar arbitrary finite group $G$. We also write $\mathbb{Q}_{n}$ to denote the cyclotomic field $\mathbb{Q}\left[e^{2 \pi i / n}\right]$ We know that $\mathbb{Q}(\chi) \subseteq \mathbb{Q}_{|G|}$, and we define $f(\chi)$ to be the smallest positive intege 
$n$ such that $\mathbb{Q}(\chi) \subseteq \mathbb{Q}_{n}$. Observe that if also $\mathbb{Q}(\chi) \subseteq \mathbb{Q}_{m}$ for some integer $m$, then in fact $n$ must divide $m$. This is so because $\mathbb{Q}_{n} \cap \mathbb{Q}_{m}=\mathbb{Q}_{d}$, where $d=\operatorname{gcd}(m, n)$, and since $d \leq n=f(\chi)$, we conclude that $d=n$, and thus $n$ divides $m$, as claimed. Feit's conjecture is that whenever $\chi \in \operatorname{Irr}(G)$, the group $G$ necessarily contains an element of order $f(\chi)$.

The first proof of the solvable case of Feit's conjecture was given by G. Amit and D. Chillag in [1], and shortly thereafter, a proof via character factorization theory was found by P. Ferguson and A. Turull [2]. As a demonstration of the power of factorization techniques in the character theory of solvable groups, we present in this section a slight variation on the Ferguson-Turull argument.

Theorem 2.7 allows us to deduce that the values of $\pi$-special characters look just like the values of irreducible characters of $\pi$-groups. (This is consistent with the point of view that a $\pi$-special character of $G$ thinks that $G$ is a $\pi$-group.) Specifically, we have the following.

LEMMA 3.1. Let $\alpha \in \operatorname{Irr}(G)$ be $\pi$-special. Then the field $\mathbb{Q}(\alpha)$ is contained in $\mathbb{Q}[\epsilon]$, where $\epsilon$ is an nth root of unity for some $\pi$-number $n$. More precisely, we have $\mathbb{Q}(\alpha)=\mathbb{Q}\left(\alpha_{H}\right)$, where $H$ is a Hall $\pi$-subgroup of $G$.

PROOF. It suffices to prove the last assertion, and since it is obvious that $\mathbb{Q}\left(\alpha_{H}\right) \subseteq$ $\mathbb{Q}(\alpha)$, it is enough to show that this field extension, which is necessarily a Galois extension, has trivial Galois group. Suppose that $\sigma \in \operatorname{Gal}\left(\mathbb{Q}(\alpha) / \mathbb{Q}\left(\alpha_{H}\right)\right)$. Observe that $\alpha^{\sigma} \in \operatorname{Irr}(G)$, and that in fact, $\alpha^{\sigma}$ is $\pi$-special. (This is because field automorphisms preserve both degrees and determinantal orders of characters.) We have

$$
\alpha_{H}=\left(\alpha_{H}\right)^{\sigma}=\left(\alpha^{\sigma}\right)_{H},
$$

and thus $\alpha=\alpha^{\sigma}$ by the injectivity of the restriction map in Theorem 2.7. It follows that all values of $\alpha$ are fixed by $\sigma$, and thus $\sigma$ is the identity automorphism of $\mathbb{Q}(\alpha)$, as required.

A similar argument yields the following.

LEMMA 3.2. Let $\chi=\alpha \beta$, where $\alpha$ and $\beta$ are respectively $\pi$-special and $\pi^{\prime}$-special characters of a $\pi$-separable group $G$. Then $\mathbb{Q}(\alpha) \subseteq \mathbb{Q}(\chi)$.

Proof. We certainly have $\mathbb{Q}(\chi) \subseteq \mathbb{Q}(\alpha, \beta)$, and this is a Galois extension of fields. It suffices to show that any automorphism $\sigma$ of $\mathbb{Q}(\alpha, \beta)$ that fixes $\chi$ also fixes $\alpha$. This holds because $\chi=\chi^{\sigma}=\alpha^{\sigma} \beta^{\sigma}$, and $\alpha^{\sigma}$ and $\beta^{\sigma}$ are respectively $\pi$-special and $\pi^{\prime}$-special. Thus $\alpha^{\sigma}=\alpha$ by the uniqueness in the Gajendragadkar factorization. 
The first step in the proof of the solvable case of Feit's conjecture is to observe that we need consider only primitive characters.

LEMMA 3.3. Suppose that $H \subseteq G$ and that $\chi \in \operatorname{Irr}(G)$ has the form $\chi=\psi^{G}$ for some character $\psi \in \operatorname{Irr}(H)$. Then $f(\chi)$ divides $f(\psi)$. In particular, if Feit's conjecture holds in $H$, it also holds in $G$.

Proof. Certainly $\mathbb{Q}(\chi) \subseteq \mathbb{Q}(\psi) \subseteq \mathbb{Q}_{f(\psi)}$, and thus $f(\chi)$ divides $f(\psi)$, as required. If $H$ contains an element $x$ of order $f(\psi)$, then some power of $x$ is the desired element of $G$ with order $f(\chi)$.

Suppose now that $f(\chi)=n$, where $\chi \in \operatorname{Irr}(G)$. For each prime divisor $p$ of $n$, let $\sigma_{p}$ denote a generator of the cyclic Galois group $\operatorname{Gal}\left(\mathbb{Q}_{n} / \mathbb{Q}_{n / p}\right)$, and observe that $\chi$ is not invariant under $\sigma_{p}$ since $\mathbb{Q}_{n / p}$ does not contain all values of $\chi$. Assuming that $G$ is solvable, and supposing (as we may) that $\chi$ is primitive, we can factor $\chi=\alpha_{p} \beta_{p}$, where $\alpha_{p}$ is $p$-special and $\beta_{p}$ is $p^{\prime}$-special. By Lemmas 3.1 and 3.2, we deduce that $\mathbb{Q}\left(\beta_{p}\right) \subseteq \mathbb{Q}(\chi) \cap \mathbb{Q}_{m}$, where $m$ is the $p^{\prime}$-part of $|G|$. Thus $\mathbb{Q}\left(\beta_{p}\right) \subseteq \mathbb{Q}_{n} \cap \mathbb{Q}_{m} \subseteq \mathbb{Q}_{n / p}$, and hence $\beta_{p}$ is fixed by $\sigma_{p}$. Since $\chi$ is not invariant under this field automorphism and $\mathbb{Q}\left(\alpha_{p}\right) \subseteq \mathbb{Q}(\chi)$, we conclude that $\left(\alpha_{p}\right)^{\sigma_{p}}$ is defined and is unequal to $\alpha_{p}$.

Consider now the function

$$
\psi=\prod_{p \mid n}\left(\alpha_{p}-\left(\alpha_{p}\right)^{\sigma_{p}}\right) .
$$

Expansion of the product shows that $\psi$ can be expressed as a sum and difference of characters, each of which is a product of the form $\prod_{p} \gamma_{p}$, where for each prime $p \mid n$, the character $\gamma_{p}$ is one of $\alpha_{p}$ or $\left(\alpha_{p}\right)^{\sigma_{p}}$. In either case, $\gamma_{p}$ is $p$-special, and thus by Theorem 2.10, each of these summands of $\psi$ is irreducible and they are all distinct. It follows that there can be no cancellation, and in particular, $\psi$ is nonzero.

Now let $x \in G$ be any element for which $\psi(x) \neq 0$. The proof of the solvable case of Feit's conjecture will be complete if we can show that the order o $(x)$ of $x$ is a multiple of $n$. For each prime divisor $p$ of $n$, we know that $\left(\alpha_{p}-\left(\alpha_{p}\right)^{\sigma_{p}}\right)(x) \neq 0$, and hence $\alpha_{p}(x)$ is a member of the field $\mathbb{Q}_{n}$ that is not invariant under $\sigma_{p}$. This number does not, therefore, lie in $\mathbb{Q}_{n / p}$. Since $\alpha_{p}(x)$ certainly lies in $\mathbb{Q}_{\mathrm{o}(x)}$, it follows that the full $p$-part of $n$ must divide o $(x)$. But holds for all prime divisors of $n$, and thus $n$ divides $\mathrm{o}(x)$, as desired.

\section{The nucleus}

Since an irreducible character of a group $G$ can always be written in the form $\psi^{G}$ for some primitive character $\psi$ of some subgroup $H \subseteq G$, it follows by Theorem 2.4 
that if $G$ is $\pi$-separable, then every irreducible character is induced from some $\pi$ factored character. The pair $(H, \psi)$ is certainly not uniquely determined (not even up to conjugacy) by the conditions that $\psi$ is a primitive character of the subgroup $H$ and that $\psi^{G}=\chi$. Even without uniqueness, one might hope that there is some set of additional requirements or some particular construction that can be used to determine a canonical choice of the $G$-conjugacy class of the pair $(H, \psi)$.

If there is a canonical way to define a 'primitive inducing pair' $(H, \psi)$ for a character $\chi \in \operatorname{Irr}(G)$, it is unknown to this author. Nevertheless, in a $\pi$-separable group $G$, it is possible to construct a pair $(W, \gamma)$, uniquely determined up to conjugacy in $G$, where $W \subseteq G$ and $\gamma \in \operatorname{Irr}(W)$ is $\pi$-factored and satisfies $\gamma^{G}=\chi$. We call a pair obtained in this way a 'nucleus' for $\chi$, and it is the purpose of this section to discuss the construction of nuclei. (The details all appear in [8].)

We start by considering the collection of all ordered pairs $(H, \xi)$, where $H \subseteq G$ and $\xi \in \operatorname{Irr}(H)$. Observe that $G$ acts on the set of such pairs by $(H, \xi)^{g}=\left(H^{g}, \xi^{g}\right)$, where $\xi^{g} \in \operatorname{Irr}\left(H^{g}\right)$ is, of course, defined by the formula $\xi^{g}\left(h^{8}\right)=\xi(h)$. Also, we construct a partial order on the set of pairs by defining $(H, \xi) \leq(K, \eta)$ if $H \subseteq K$ and $\xi$ is a constituent of the restriction $\eta_{H}$.

Assuming now that $G$ is $\pi$-separable, we let $\mathscr{F}=\mathscr{F}(G)$ be the set of subnormal $\pi$-factored pairs. These are the pairs $(S, \theta)$ for which $S \triangleleft \triangleleft G$ and $\theta \in \operatorname{Irr}(S)$ is $\pi$-factored. We are particularly interested in the subset of $\mathscr{F}$ consisting of all pairs maximal in $\mathscr{F}$, and we write $\mathscr{F}^{*}$ to denote this set of maximal subnormal $\pi$-factored pairs.

If $\chi \in \operatorname{Irr}(G)$, then it is obvious that there exist some pairs $(S, \theta) \in \mathscr{F}$ such that $(S, \theta) \leq(G, \chi)$. Of course, there will exist pairs in $\mathscr{F}$ maximal with this property, but perhaps it is not so obvious that there actually exist pairs in $\mathscr{F}^{*}$ which lie below $(G, \chi)$.

THEOREM 4.1. Let $G$ be $\pi$-separable and suppose $\chi \in \operatorname{Irr}(G)$. Then there exists $(S, \theta) \in \mathscr{F}^{*}(G)$ such that $(S, \theta) \leq(G, \chi)$. Furthermore, the pair $(S, \theta)$ is uniquely determined up to conjugation by elements of $G$.

Each character $\chi \in \operatorname{Irr}(G)$ determines via Theorem 4.1 a particular $G$-orbit in $\mathscr{F}^{*}$, and it is natural to consider the stabilizer $T=\mathbf{N}_{G}((S, \theta))$ of a member $(S, \theta)$ of this orbit. If we knew that $S$ was normal in $G$ (and not merely subnormal), then $T$ would be the inertia group of the character $\theta$ of $S$, and the Clifford correspondence would apply. In particular, there would exist a unique irreducible character $\psi \in \operatorname{Irr}(T)$ lying over $\theta$ and such that $\psi^{G}=\chi$. Remarkably, this holds even without assuming that $S$ is normal.

THEOREM 4.2. Assume that $G$ is $\pi$-separable. Let $(S, \theta) \in \mathscr{F}^{*}(G)$, and write $T=\mathbf{N}_{G}((S, \theta))$. Then induction defines a bijection from $\operatorname{Irr}(T \mid \theta)$ onto $\operatorname{Irr}(G \mid \theta)$. 
Given $\chi \in \operatorname{Irr}(G)$, it is obvious that if $\chi$ is $\pi$-factored, then the pair $(S, \theta)$ given by Theorem 4.1 is just $(G, \chi)$. Otherwise, $S$ is a proper subgroup of $G$. (In fact, one could view the index of $S$, or perhaps the subnormal depth of $S$, as a measure of how far $\chi$ is from being $\pi$-factored.) It is perhaps not completely obvious that if $\chi$ is not a factored character, then not only is $S<G$, but also $T<G$, where $T=\mathbf{N}_{G}((S, \theta))$, as before. To see this, write $\theta=\alpha \beta$, where $\alpha$ and $\beta$ are respectively $\pi$-special and $\pi^{\prime}$-special. Note that we can write $T=A \cap B$, where $A=\mathbf{N}_{G}((S, \alpha))$ and $B=\mathbf{N}_{G}((S, \beta))$. Note also that both $A$ and $B$ are contained in $N=\mathbf{N}_{G}(S)$.

LEMMA 4.3. Assume the previous notation and suppose that $\chi \in \operatorname{Irr}(G)$ is not $\pi$-factored. Then either $|N: B|$ is divisible by some prime in $\pi$ or $|N: A|$ is divisible by some prime in $\pi^{\prime}$. In particular, $T=A \cap B<G$.

PROOF. Since $\chi$ is not $\pi$-factored, we know that $S<G$, and we can choose $U \triangleleft \triangleleft G$ such that $U / S$ is a composition factor of $G$. Since $U>S$ and $(S, \theta) \in \mathscr{F}^{*}$, we know that no irreducible constituent of $\theta^{U}$ can be $\pi$-factored.

If $U / S$ is a $\pi$-group, then by Lemma 2.5 we see that $\beta$ is not invariant in $U$, and hence $U \nsubseteq B$. Since $U / S$ is a subnormal $\pi$-subgroup of $N / S$ that is not contained in $B / S$, it follows that the index $|N: B|$ is divisible by some prime in $\pi$. Similarly, if $U / S$ is a $\pi^{\prime}$-group, then $|N: A|$ is divisible by some prime in $\pi^{\prime}$.

We are now ready to define the nucleus of an arbitrary irreducible character of our $\pi$-separable group $G$. If $\chi \in \operatorname{Irr}(G)$ is not $\pi$-factored, it follows from the previous three results that there exists a pair $(T, \psi)$, where $T<G$ is the stabilizer in $G$ of some member of $\mathscr{F}^{*}$ and $\psi^{G}=\chi$. Furthermore, the pair $(T, \psi)$ (which we call a standard inducing pair for $\chi$ ) is uniquely determined up to $G$-conjugacy. If $\psi$ is $\pi$-factored, stop; otherwise construct a standard inducing pair for $\psi$. Continuing like this, we see that the process must eventually stop, since at each stage we get a proper subgroup. At the termination of this iterative procedure, we have a pair $(W, \gamma)$, where $\gamma$ is a $\pi$-factored irreducible character of $W$. Furthermore, $(W, \gamma)$ is uniquely determined by $\chi$ up to $G$-conjugacy. We write nuc $(\chi)$ to denote the $G$-orbit of pairs containing $(W, \gamma)$, and we refer to each member of this orbit as a nucleus for $\chi$. Specifically, we say that $W$ is a nucleus subgroup for $\chi$ and that $\gamma$ is a nucleus character for $\chi$.

\section{Partial characters}

As usual, we have in mind a fixed set $\pi$ of prime numbers. For any group $G$, we write $G^{0}$ to denote the set of $\pi$-elements of $G$, the subset consisting of all elements whose order involves only primes in $\pi$. Also, for any (complex valued) class function 
$\varphi$ of $G$, we write $\varphi^{0}$ to denote the restriction of $\varphi$ to $G^{0}$. The map $\varphi \mapsto \varphi^{0}$ is clearly a complex vector space homomorphism which maps the space $\operatorname{cf}(G)$ of class functions of $G$ onto $\operatorname{cf}(G)^{0}$, the space of $\pi$-class functions, the functions defined on $G^{0}$ and constant on conjugacy classes.

If $\chi$ is a character of $G$, we say that the $\pi$-class function $\chi^{0}$ is a $\pi$-partial character of $G$. The motivation for this definition is the 'classical' case where $\pi=p^{\prime}$, the complement of a prime number $p$. In this case, the Brauer characters of $G$ are defined only on $G^{0}$, and it is a result of Brauer that every $p^{\prime}$-partial character is a (possibly reducible) Brauer character. Furthermore, if $G$ happens to be $\pi$-separable (which is the same as $p$-solvable in this situation) the Fong-Swan theorem guarantees that every irreducible Brauer character of $G$ is a $\pi$-partial character. It follows that when $\pi=p^{\prime}$ and $G$ is $\pi$-separable, the $\pi$-partial characters of $G$ are exactly the Brauer characters of $G$. Our goal is to generalize the theory of Brauer characters for arbitrary prime sets $\pi$, for $\pi$-separable groups. We do this by studying the $\pi$-partial characters of $G$ rather than by trying to find some $\pi$-analog of modular representation theory.

Actually, some care is needed here. If $N$ is the subgroup of $G$ generated by the $\pi$ elements, it is certainly possible to have $N<G$. In this case $N^{0}=G^{0}$ and there exist characters $\chi$ of $G$ and $\psi$ of $N$ having identical restrictions to $G^{0}=N^{0}$. Nevertheless, we should not consider $\chi^{0}$ and $\psi^{0}$ to be the same $\pi$-partial character; one should be viewed as a $\pi$-partial character of $G$ and the other as a $\pi$-partial character of $N$. (In the case of Brauer characters, for example, if $N=\mathbf{O}^{p}(G)$, and $\varphi$ is a Brauer character of $G$, we would not consider the Brauer character $\varphi_{N}$ to be the same object as $\varphi$, even though they are they are functions with equal domains and equal values. It is entirely possible, for example, that $\varphi$ is an irreducible Brauer character of $G$ and yet $\varphi_{N}$ is reducible when viewed as a Brauer character of $N$.) We want $\pi$-partial characters, therefore, to retain some 'memory' of their original domain of definition. If we were willing to be somewhat pedantic, we might have defined a $\pi$-partial character of $G$ to be an ordered pair whose first component is a function defined on $G^{0}$ and whose second component is the group $G$.

Since the set $\operatorname{Irr}(G)$ spans the vector space $\operatorname{cf}(G)$ over $\mathbb{C}$, it is immediate that the $\pi$-partial characters of an arbitrary group $G \operatorname{span} \operatorname{cf}(G)^{0}$. In the case where $\pi=p^{\prime}$, the set $\operatorname{IBr}(G)$ is a basis for $\operatorname{cf}(G)^{0}$, and if $G$ is $p$-solvable, this is a basis consisting of $\pi$-partial characters. Furthermore, every $\pi$-partial character can be expressed as a nonnegative integer linear combination of members of this basis.

We restrict our attention now to $\pi$-separable groups $G$, where $\pi$ is an arbitrary prime set. It would be pleasant if we could find in this situation (as we can when $\left.\pi=p^{\prime}\right)$ a basis for $\operatorname{cf}(G)^{0}$ consisting of $\pi$-partial characters and having the additional property that every $\pi$-partial character of $G$ is a nonnegative integer combination of this basis. We seek, in other words, some sort of $\pi$-analog of irreducible Brauer characters in $\pi$-separable groups. 
It is a fact (as we shall see later) that in the case where $\pi=p^{\prime}$, the characters $\chi^{0}$ lie in $\operatorname{IBr}(G)$ for all $p^{\prime}$-special characters $\chi$ of $G$. In this situation, however, the only Brauer characters that could possibly be restrictions of $p^{\prime}$-special characters are those having $p^{\prime}$-degree, and these usually do not exhaust the set $\operatorname{IBr}(G)$. This suggests that in the general case (where $\pi$ is arbitrary), we might be able to use the $\pi$-partial characters $\chi^{0}$, where $\chi$ is $\pi$-special, as at least a part of the basis we seek for $\operatorname{cf}(G)^{0}$.

By Theorem 2.7 we know that the restrictions of the $\pi$-special characters of $G$ to a Hall $\pi$-subgroup $H$ are distinct irreducible characters of $H$, and thus they are linearly independent functions on $H$. Since $H \subseteq G^{0}$, it follows that the $\pi$-partial characters $\chi^{0}$ are distinct and linearly independent as $\chi$ runs over $\mathscr{X}_{\pi}(G)$, the set of $\pi$-special characters of $G$. Our task now is to find some natural way to expand this set to a full basis for $\operatorname{cf}(G)^{0}$. In particular, we would like to expand the set $\mathscr{X}_{\pi}(G)$ by somehow weakening the eligibility requirement that character degrees must be $\pi$-numbers.

It is a theorem of $\mathrm{B}$. Huppert that in a $p$-solvable group, every irreducible Brauer character is induced from a Brauer character of $p^{\prime}$-degree of some subgroup. This suggests that it might be possible to obtain the desired expansion of the set of $\pi$-special characters by including certain characters that are induced from $\pi$-special characters of subgroups. We would like to do this in such a way that the resulting characters are still intimately associated with the prime set $\pi$, even though they might not have $\pi$-degree.

\section{The set $\mathrm{B}_{\pi}(G)$}

We are now ready to define the character set $\mathrm{B}_{\pi}(G)$ for a $\pi$-separable group $G$. This is the set of those irreducible characters of $G$ for which a nucleus character is $\pi$-special. Since a $\pi$-factored character is always its own nucleus character, we see that the $\pi$-special characters of $G$ all lie in $\mathrm{B}_{\pi}(G)$, and they are exactly the $\pi$-factored characters in this set.

It is also true that the $\pi$-special characters of $G$ are exactly those members of $\mathrm{B}_{\pi}(G)$ which have $\pi$-degree.

LEMMA 6.1. Suppose that $\chi(1)$ is a $\pi$-number, where $\chi \in \mathrm{B}_{\pi}(G)$. Then $\chi$ is $\pi$-special.

ProOF. Let $(W, \gamma)$ be a nucleus for $\chi \in \mathrm{B}_{\pi}(G)$. Then $\gamma$ is $\pi$-special and there exists $(S, \theta) \in \mathscr{F}^{*}(G)$ with $(S, \theta) \leq(W, \gamma)$. It follows that $\theta$ is $\pi$-special, and so in the notation of Lemma 4.3, we have $\alpha=\theta$ and $A=T$. Also, $\beta$ is the trivial character of $S$ and $B=N$, and thus $|N: B|$ is divisible by no prime in $\pi$. Furthermore, since $\chi$ has $\pi$-degree and is induced from $T$, we see that $|G: T|$ is a $\pi$-number, and thus $|N: A|$ is divisible by no prime in $\pi^{\prime}$. By Lemma 4.3 , therefore, it follows that $\chi$ is $\pi$-factored, and since it lies in $\mathrm{B}_{\pi}(G)$, we deduce that it is $\pi$-special. 
The characters in $\mathrm{B}_{\pi}(G)$ can be viewed as being 'generalized' $\pi$-special, and in a number of ways, their behavior is analogous to that of genuine $\pi$-special characters. The principal exception to this, of course, is that they need not have $\pi$-degree. It is true, for example, although it is certainly not obvious, that the property of being a $\mathrm{B}_{\pi}$-character is normally heritable, and there is also a strong analog for Lemma 2.6 .

THEOREM 6.2. Let $N \triangleleft G$, where $G$ is $\pi$-separable, and suppose $\chi \in \mathrm{B}_{\pi}(G)$. Then all irreducible constituents of $\chi_{N}$ lie in $\mathrm{B}_{\pi}(N)$.

THEOREM 6.3. Let $N \triangleleft G$, where $G$ is $\pi$-separable, and suppose $\psi \in \mathrm{B}_{\pi}(N)$.

(a) If $G / N$ is a $\pi$-group, then every character $\chi \in \operatorname{Irr}(G)$ that lies over $\psi$ is in $\mathrm{B}_{\pi}(G)$.

(b) If $G / N$ is a $\pi^{\prime}$-group, then there is a unique character $\chi \in \mathrm{B}_{\pi}(G)$ which lies over $\psi$. In this case, $\left[\chi_{N}, \psi\right]=1$.

The proofs of Theorems 6.2 and 6.3 and of all the results in this section can be found in [8].

Observe that in contrast with Lemma 2.6(b), it is not assumed in 6.3(b) that the character $\psi$ is invariant in $G$. Because of this, the degree of the character $\chi$ may have a larger $\pi^{\prime}$-part than does the degree of $\psi$, but since eligibility for membership in the set $\mathrm{B}_{\pi}(G)$ imposes no requirement on the degree of a character, this is not a problem. If it happens that $\psi$ is invariant in $G$, however, the last part of assertion 6.3(b) guarantees that $\chi$ is an extension of $\psi$, and the situation is entirely analogous to that in Lemma 2.6.

In many cases, Theorem 6.3 (together with the fact that $\pi$-special characters are $\mathrm{B}_{\pi}$-characters) suffices to determine the set $\mathrm{B}_{\pi}(G)$. This is significant because in practice, it is difficult to compute a nucleus for a character, and so it is awkward to try to use the definition of the set $\mathrm{B}_{\pi}(G)$ in order to test membership.

We can use Theorem 6.3 in the case where $G$ is the symmetric group $S_{4}$, for example, to compute the sets $\mathrm{B}_{\{2\}}(G)$ and $\mathrm{B}_{\{3\}}(G)$. Writing $K$ to denote the Klein subgroup of order 4 and $A$ to denote the alternating group $A_{4}$, we see that all four linear characters of $K$ are $\{2\}$-special, and hence lie in $\mathrm{B}_{12\}}(K)$. It follows from Theorem 6.3 that the irreducible character of $A$ of degree 3 lies in $\mathrm{B}_{\{2\}}(A)$. Of the three linear characters of $A$, only the principal character is $\{2\}$-special and can lie in $\mathrm{B}_{\{2\}}(A)$. We now see by Theorem 6.3 that $\mathrm{B}_{\{2\}}(G)$ consists exactly of the two irreducible characters of $G$ of degree 3 and the two linear characters.

To compute $\mathrm{B}_{\{3\}}(G)$, we start with the observation that only the principal character of $K$ lies in $\mathrm{B}_{\{3\}}(K)$, and thus $\mathrm{B}_{\{3\}}(A)$ consists exactly of the three linear characters of $A$. It then follows that the principal character and the irreducible character of degree 2 are exactly the members of $\mathrm{B}_{\{3\}}(G)$.

Recall that by Theorem 2.7, restriction defines an injection from the set $\mathscr{X}_{\pi}(G)$ 
of $\pi$-special characters of $G$ into $\operatorname{Irr}(H)$, where $H$ is a Hall $\pi$-subgroup of $G$. We consider next the generalization of this result for the set $\mathrm{B}_{\pi}(G)$.

THEOREM 6.4. Let $H$ be a Hall $\pi$-subgroup of the $\pi$-separable group $G$ and suppose that $\chi \in \mathrm{B}_{\pi}(G)$. Let $\alpha$ be any irreducible constituent of $\chi_{H}$ of smallest possible degree. The following then hold.

(a) The degree $\alpha(1)$ is exactly the $\pi$-part $\chi(1)_{\pi}$ of $\chi(1)$.

(b) The multiplicity of $\alpha$ as a constituent of $\chi_{H}$ is 1 .

(c) If $\chi \neq \psi \in \mathrm{B}_{\pi}(G)$, then $\alpha$ does not occur as a constituent of $\psi_{H}$.

Observe that if $\chi \in \operatorname{Irr}(G)$ is $\pi$-special, then Theorem 6.4(a) tells us that $\chi_{H}=\alpha$ is irreducible, and by Theorem 6.4(c), no other $\pi$-special character of $G$ has the same restriction to $H$. In other words, Gajendragadkar's Theorem 2.7 is included in Theorem 6.4.

If $H$ is a Hall $\pi$-subgroup of $G$ as in Theorem 6.4, we say that a character $\alpha \in \operatorname{Irr}(H)$ is a Fong character in $G$ if it occurs as a constituent of minimal degree in $\chi_{H}$ for some character $\chi \in \mathrm{B}_{\pi}(G)$. By Theorem 6.4(c), the Fong character $\alpha$ uniquely determines $\chi$, and we say that $\alpha$ is a Fong character belonging to, or associated with $\chi$. We stress that for each character $\chi \in \mathrm{B}_{\pi}(G)$, there may be several different associated Fong characters, and it should be clear that these are permuted by the group $\mathbf{N}_{G}(H)$. Unfortunately, there can be more than one orbit of Fong characters belonging to $\chi$ under this action.

We mention that we have chosen the name 'Fong characters' for these irreducible constituents of minimal degree of the restrictions of $B_{\pi}$-characters to Hall $\pi$-subgroups because in the 'classical' case, where $\pi$ is the complement of a prime, there is a result of P. Fong that is very closely related to our Theorem 6.4. (See Theorems $2 B$ and 2D of [3].)

Although we will not discuss the details of the proof of Theorem 6.4 here, we can present some of the ideas that are involved.

LEMMA 6.5. Let $H$ be a Hall $\pi$-subgroup of a $\pi$-separable group $G$. If $\chi \in \mathrm{B}_{\pi}(G)$, then $\chi_{H}$ has an irreducible constituent with degree not exceeding $\chi(1)_{\pi}$.

PROOF. Write $\chi=\gamma^{G}$, where $\gamma$ is an irreducible character of $\pi$-degree of some subgroup $W \subseteq G$. Observe that the required pair $(W, \gamma)$ exists, since we could take it to be a nucleus for $\chi$. Replacing $(W, \gamma)$ by a conjugate if necessary, we may assume that $H \cap W$ is a Hall $\pi$-subgroup of $W$.

Now define $\alpha=\left(\gamma_{(H \cap W)}\right)^{H}$ and observe that $\alpha(1)=\gamma(1)|H: H \cap W|$ is a $\pi$-number. Now $\chi(1)=\gamma(1)|G: W|$, and so we compute that

$$
\alpha(1)=|H: H \cap W| \gamma(1)=\frac{|H: H \cap W|}{|G: W|} \chi(1) .
$$


Thus

$$
|G: H| \alpha(1)=|W: H \cap W| \chi(1),
$$

and taking $\pi$-parts of both sides, we see that $\alpha(1)=\chi(1)_{\pi}$.

By Mackey's induction theorem, we know that $\alpha$ is a constituent of $\chi_{H}$, and it follows that every irreducible constituent of $\alpha$ satisfies the conclusion of the lemma.

After Theorem 6.4(a) is proved, we can deduce that the character $\alpha$ in the above argument must be irreducible, and it is thus a Fong character belonging to $\chi$. We note also that if $\chi$ is a monomial character, we can take $\gamma$ to be linear in the proof of Lemma 6.5. It follows in this case that $\alpha$ is monomial, and we record this observation here for future reference.

COROLLARY 6.6. Suppose that $\chi \in \mathrm{B}_{\pi}(G)$ is monomial. Then some Fong character belonging to $\chi$ is monomial.

The following result, when combined with Lemma 6.5 , easily yields Theorem 6.4 . We mention this theorem (although we do not give the proof) because it is interesting that it applies to all irreducible characters of a Hall $\pi$-subgroup, and not just to Fong characters. This greater generality has not yet been exploited.

THEOREM 6.7. Let $H$ be a Hall $\pi$-subgroup of the $\pi$-separable group $G$. If $\alpha \in$ $\operatorname{Irr}(H)$, then $\alpha(1)$ is equal to the sum (counting multiplicities) of the $\pi$-parts of those irreducible constituents of $\alpha^{G}$ which lie in $\mathrm{B}_{\pi}(G)$. In other words,

$$
\alpha(1)=\sum_{\chi \in \mathrm{B}_{\pi}(G)}\left[\alpha^{\mathrm{G}}, \chi\right] \chi(1)_{\pi} .
$$

\section{Irreducible partial characters}

If $G$ is $p$-solvable, we know by the Fong-Swan theorem that the set $\operatorname{IBr}(G)$ of irreducible Brauer characters at the prime $p$ is a set of $\pi$-partial characters of $G$, where $\pi=p^{\prime}$. We are now ready to construct the set $\mathrm{I}_{\pi}(G)$, which turns out to be the analog of $\operatorname{IBr}(G)$ for $\pi$-separable groups $G$, where $\pi$ is an arbitrary prime set.

We define $\mathrm{I}_{\pi}(G)$ simply to be the set of $\pi$-partial characters $\chi^{0}$ for $\chi \in \mathrm{B}_{\pi}(G)$.

LEMMA 7.1. The set $\mathrm{I}_{\pi}(G)$ is linearly independent and the map $\chi \mapsto \chi^{0}$ from $\mathrm{B}_{\pi}(G)$ onto $\mathrm{I}_{\pi}(G)$ is injective.

ProOf. Let $H$ be a Hall $\pi$-subgroup of $G$ and note that $H \subseteq G^{0}$. It suffices to show that if

$$
\sum_{\chi \in \mathrm{B}_{\pi}(G)} a_{\chi} \chi_{H}=0
$$


with complex coefficients $a_{\chi}$, then $a_{\chi}=0$ for all $\chi \in \mathrm{B}_{\pi}(G)$. This follows from Theorem 6.4(c), since Fong characters belonging to different members of $\mathrm{B}_{\pi}(G)$ are different, and they cannot cancel.

In fact, we have the following.

THEOREM 7.2. If $G$ is $\pi$-separable, then $\mathrm{I}_{\pi}(G)$ is a basis for the $\operatorname{set} \operatorname{cf}(G)^{0}$ of $\pi$-class functions of $G$.

What is needed to complete the proof of this theorem is to count the members of $\mathrm{B}_{\pi}(G)$. Specifically, we need to show that the number of characters in this set is exactly equal to the number of $\pi$-classes of $G$. The proof of this proceeds by induction on $|G|$, and we sketch part of the argument.

Let $N$ be a maximal normal subgroup of $G$. By the inductive hypothesis (and Lemma 7.1) it follows that the table of values of characters in $\mathrm{B}_{\pi}(N)$ at $\pi$-classes of $N$ is a square invertible matrix. It follows by Brauer's permutation lemma that the number of $G$-orbits of $\pi$-classes of $N$ is equal to the number of $G$-orbits of $\mathrm{B}_{\pi}$ characters of $N$. The first of these numbers is just the count of those $\pi$-classes of $G$ that happen to be contained in $N$. In the case where $G / N$ is a $\pi^{\prime}$-group, these are, of course, all of the $\pi$-classes of $G$. Also in this case, it follows by Theorem 6.3 that there is exactly one character in $\mathrm{B}_{\pi}(G)$ lying over each $G$-orbit of $\mathrm{B}_{\pi}(N)$, and by Theorem 6.2, this accounts for all members of $\mathrm{B}_{\pi}(G)$. The result thus follows when $G / N$ is a $\pi^{\prime}$-group. The proof in the remaining case, where $G / N$ is a $\pi$-group, is technically more difficult, and it appears it [8]. We will not discuss it further here.

Not only is $\mathrm{I}_{\pi}(G)$ a basis for $\operatorname{cf}(G)^{0}$; as promised, even more is true.

COROLLARY 7.3. Every $\pi$-partial character of $G$ is a nonnegative integer linear combination of $\mathrm{I}_{\pi}(G)$.

PROOF. Let $\psi \in \operatorname{Char}(G)$ and write

$$
\psi^{0}=\sum_{\varphi \in \mathrm{I}_{\pi}(G)} a_{\varphi} \varphi
$$

with complex coefficients $a_{\varphi}$. Let $\chi_{\varphi}$ be the unique character in $\mathrm{B}_{\pi}(G)$ whose restriction to $G^{0}$ is $\varphi$, and choose some Fong character $\alpha_{\varphi}$ belonging to $\chi_{\varphi}$. (We assume that we have fixed some Hall $\pi$-subgroup $H$ of $G$ and that all of the Fong characters $\alpha_{\varphi}$ lie in $\operatorname{Irr}(H)$.)

By Theorem 6.4(b), the character $\alpha_{\varphi}$ occurs with multiplicity 1 in $\varphi_{H}$, and by Theorem 6.4(c) it does not occur at all as a constituent of $\theta_{H}$ if $\varphi \neq \theta \in \mathrm{I}_{\pi}(G)$. It follows that $a_{\varphi}$ is exactly the multiplicity of $\alpha_{\varphi}$ as a constituent of the character $\psi_{H}$. It is therefore a nonnegative integer, as claimed. 
It is immediate from Corollary 7.3 that $\mathrm{I}_{\pi}(G)$ is exactly the set of irreducible $\pi$-partial characters of $G$, those which cannot be written as sums of other $\pi$-partial characters. It follows that the set $\mathrm{I}_{\pi}(G)$ can be constructed without first locating the (somewhat elusive) $\mathbf{B}_{\pi}$-characters. One can read off $\mathrm{I}_{\pi}(G)$ directly from the character table of $G$ simply by deleting all columns that do not correspond to $\pi$-classes, and then deleting (one-by-one) rows of the resulting matrix which can be written as nonnegative integer combinations of the remaining rows. We know that this process will terminate with the square table of values of $\mathrm{I}_{\pi}(G)$.

There is available a direct proof, independent of the theory of nuclei and of the definition of $\mathrm{B}_{\pi}(G)$, that the process for the construction of $\mathrm{I}_{\pi}(G)$ described in the previous paragraph necessarily results in a basis for $\mathrm{cf}(G)^{0}$. This argument, originally due to G. Robinson and R. Staszewski [11], appears in somewhat simplified form in [10].

The definition and many of the properties of Fong characters are also independent of the construction of $\mathrm{B}_{\pi}(G)$. If $\varphi \in \mathrm{I}_{\pi}(G)$, then $\varphi_{H}=\chi_{H}$, where $H$ is a Hall $\pi$ subgroup and $\chi \in \mathrm{B}_{\pi}(G)$ satisfies $\chi^{0}=\varphi$. In particular, $\varphi_{H}$ is an ordinary character of $H$, and its irreducible constituents of least degree can be described as the Fong characters belonging to $\varphi$. A version of Theorem 6.4 can thus be stated with $\mathrm{I}_{\pi}(G)$ in place of $\mathrm{B}_{\pi}(G)$, and this is proved in [10] without appeal to the theory of nuclei or to the definition of $\mathrm{B}_{\pi}(G)$.

Unfortunately, some of the properties of the irreducible $\pi$-partial characters that are needed for applications seem to be unobtainable without going through the full development of $\pi$-theory as we have outlined it here. It follows from our results, for example, that if $\varphi \in \mathrm{I}_{\pi}(G)$ and $N \triangleleft G$, then $\varphi_{N}$ is a multiple of the sum of the members of a $G$-orbit in $\mathrm{I}_{\pi}(N)$. This version of Clifford's theorem follows easily using Theorem 6.2, but it is not clear how to obtain it from the approach discussed in the previous two paragraphs.

We know from Theorem 7.2 that the number of characters in the set $\mathrm{B}_{\pi}(G)$ is equal to the number of $\pi$-classes of the $\pi$-separable group $G$. It also possible to count the $\pi$-special characters of $G$. Although we shall not need this information, we mention the result that is proved as Corollary 1.16 of [12]: the number of $\pi$-special characters of $G$ is equal to the number of $\pi$-classes in a Hall $\pi$-subgroup of the normalizer in $G$ of a $\pi$-complement of $G$.

We now prove a fact to which we alluded earlier.

COROLLARY 7.4. Let $\pi=p^{\prime}$, the complement of the prime number $p$, and consider the set $\operatorname{IBr}(G)$ of irreducible Brauer characters of $G$ at the prime $p$. If $G$ is p-solvable (that is, is $\pi$-separable), then $\operatorname{IBr}(G)=\mathrm{I}_{\pi}(G)$.

Proof. Let $\theta \in \operatorname{IBr}(G)$, and observe that by the Fong-Swan theorem, $\theta$ is a $\pi$ partial character of $G$. By Corollary 7.3, therefore, $\theta$ can be written as a nonnegative 
integer linear combination of the members of $\mathrm{I}_{\pi}(G)$. Since each of these partial characters is in fact a Brauer character, it follows from the irreducibility of $\theta$ that it is actually a member of $\mathrm{I}_{\pi}(G)$.

We now know that $\operatorname{IBr}(G) \subseteq \mathrm{I}_{\pi}(G)$, and since both sets have cardinality equal to the number of $\pi$-classes of $G$, equality holds.

In fact, something more general is proved in [8].

THEOREM 7.5. Let $G$ be $\pi$-separable, and suppose $p$ is any prime not lying in $\pi$. Then restriction to the set of p-regular elements defines an injection from $\mathrm{B}_{\pi}(G)$ into $\operatorname{IBr}(G)$, the set of irreducible Brauer characters of $G$ at the prime $p$.

Observe that $G$ is not necessarily $p$-solvable in Theorem 7.5, and so the Fong-Swan theorem is unavailable, and it is not used in the proof. Nevertheless, the theorem tells us that a certain number of the irreducible Brauer characters of the $\pi$-separable group $G$ can be lifted to ordinary characters. In the case where $\pi=p^{\prime}$, Theorem 7.5 tells us that $\mathrm{I}_{\pi}(G) \subseteq \operatorname{IBr}(G)$ and thus equality holds. In other words, this result provides an independent proof of the Fong-Swan theorem.

\section{An application}

Let $H$ be a Hall $\pi$-subgroup of a $\pi$-separable group $G$, and suppose $\psi$ is a character of $H$. We ask when it is true that there is some character $\chi$ of $G$ such that $\chi_{H}=\psi$. An obvious necessary condition is that $\psi(x)=\psi(y)$ whenever $x$ and $y$ are elements of $H$ that are conjugate in $G$. (We shall say that $\psi$ is conjugacy compatible in $G$ if this happens.) Remarkably, this condition is sufficient too.

THEOREM 8.1. Let $H \subseteq G$ be a Hall $\pi$-subgroup, where $G$ is $\pi$-separable. If $\psi \in \operatorname{Char}(H)$ is conjugacy compatible in $G$, then $\psi$ extends to a character of $G$.

Before proceeding with the proof of Theorem 8.1, we mention that without the assumption that $G$ is $\pi$-separable, the assertion would be false, even if we take $H$ to be a Sylow subgroup. In the alternating group $A_{5}$, for example, let $H$ be a Sylow 3-subgroup and take $\psi$ to be the sum of the two nonprincipal linear characters of $H$. It is trivial to check that $\psi$ is conjugacy compatible in $G$, but since the only character of $A_{5}$ of degree 2 is the sum of two copies of the principal character, we see that $\psi$ does not extend to a character of $G$ in this situation. (Note, however, that $\psi$ does extend to a generalized character in this case. This is a general phenomenon, which is easy to prove using Brauer's characterization of characters.)

We mention also that the case of Theorem 8.1 where $H$ is normal in $G$ is known and elementary. When $H$ is normal, conjugacy compatibility is equivalent to invariance in 
$G$, and if $\psi$ is irreducible, extendibility is guaranteed by Gallagher's Theorem (2.1). Even if $\psi$ is not irreducible, a routine argument suffices to reduce the problem to the situation of Theorem 2.1 .

We prove a slightly strengthened form of Theorem 8.1.

THEOREM 8.2. In the situation of Theorem 8.1 , the character $\psi$ extends to a character of $G$ all of whose irreducible constituents lie in $\mathrm{B}_{\pi}(G)$.

PROOF. Define the function $\theta \in \operatorname{cf}(G)^{0}$ as follows. If $g \in G$ is a $\pi$-element, choose $x \in H$ such that $g$ is conjugate to $x$ in $G$, and set $\theta(g)=\psi(x)$. This is well defined since if $y \in H$ had been chosen in place of $x$, with $y$ conjugate to $g$ in $G$, then $\psi(x)=\psi(y)$ by the assumption that $\psi$ is conjugacy compatible in $G$.

Now write $\theta=\sum a_{\varphi} \varphi$, where the sum runs over $\varphi \in \mathrm{I}_{\pi}(G)$ and the coefficients $a_{\varphi}$ are complex numbers. Let $\beta_{\varphi} \in \mathrm{B}_{\pi}(G)$ satisfy $\left(\beta_{\varphi}\right)^{0}=\varphi$, and choose Fong characters $\alpha_{\varphi} \in \operatorname{Irr}(H)$ associated with $\beta_{\varphi}$. By the usual argument, we see that the multiplicity of $\alpha_{\varphi}$ in $\theta_{H}$ is equal to $a_{\varphi}$. Since $\theta_{H}=\psi$ is a character of $H$, we deduce that $a_{\varphi}$ is a nonnegative integer, and it follows that $\chi=\sum a_{\varphi} \beta_{\varphi}$ is a character of $G$. Since $\left(\beta_{\varphi}\right)_{H}=\varphi_{H}$, we have $\chi_{H}=\theta_{H}=\psi$, as required, and we have shown that $\psi$ extends to a character of $G$ all of whose irreducible constituents lie in $\mathrm{B}_{\pi}(G)$.

COROLlaRY 8.3. The image of the restriction map $\mathscr{X}_{\pi}(G) \rightarrow \operatorname{Irr}(H)$ given in Theorem 2.7 is exactly the set of conjugacy compatible irreducible characters of the Hall $\pi$-subgroup $H$.

PROOF. Certainly, every character in the image of this map is conjugacy compatible in $G$, and so it suffices to prove the converse. Suppose then that $\alpha \in \operatorname{Irr}(H)$ is conjugacy compatible in $G$. By Theorem 8.2 , there is some character $\chi \in \operatorname{Char}(G)$ such that $\chi_{H}=\alpha$, and this can be done so that all irreducible constituents of $\chi$ lie in $\mathrm{B}_{\pi}(G)$.

Since $\alpha$ is irreducible, it follows that $\chi$ is irreducible, and thus $\chi \in \mathrm{B}_{\pi}(G)$. We have $\chi(1)=\alpha(1)$ is a $\pi$-number, and it follows by Lemma 6.1 that $\chi$ is $\pi$-special, as required.

\section{Fong characters}

Given a Hall subgroup $H$ of a $\pi$-separable group $G$, how can one tell whether or not a given irreducible character of $H$ happens to be a Fong character? Also, how can one tell whether or not two Fong characters of $H$ belong to the same $\mathrm{B}_{\pi}$-character of $G$ ? Answers to both questions are easy consequences of the following corollary of 7.3. 
COROLlary 9.1. Let $\psi \in \operatorname{Char}(G)$, where $G$ is $\pi$-separable, and suppose $H$ is a Hall $\pi$-subgroup of $G$. Then $\psi_{H}$ is a nonnegative integer linear combination of the characters $\chi_{H}$ for $\chi \in \mathbf{B}_{\pi}(G)$.

PROOF. We know that the $\pi$-partial character $\psi^{0}$ is a nonnegative integer linear combination of $\mathrm{I}_{\pi}(G)=\left\{\chi^{0} \mid \chi \in \mathrm{B}_{\pi}(G)\right\}$. Since $H$ is contained in $G^{0}$, we can further restrict to $H$, and the result follows.

COROLlARY 9.2. Assuming the above notation, let $\alpha \in \operatorname{Irr}(H)$. Then $\alpha$ is a Fong character of $G$ iff there is some character $\chi \in \operatorname{Irr}(G)$ such that $\alpha$ occurs as an irreducible constituent of least degree in $\chi_{H}$.

PROOF. If $\alpha$ is a Fong character belonging to $\chi \in \mathrm{B}_{\pi}(G)$, then by definition, $\alpha$ is an irreducible constituent of least degree in $\chi_{H}$.

Conversely, suppose that $\alpha$ is an irreducible constituent of least degree in $\psi_{H}$, where $\psi \in \operatorname{Irr}(G)$. By Corollary 9.1, we know that $\psi_{H}$ is a nonnegative integer linear combination of $\chi_{H}$ for $\chi \in \mathrm{B}_{\pi}(G)$, and it follows that the set of irreducible constituents of $\psi_{H}$ is the union of the sets of irreducible constituents of $\chi_{H}$, where $\chi$ runs over some subset of $\mathbf{B}_{\pi}(G)$. It follows that $\alpha$ is an irreducible constituent of least degree of $\chi_{H}$ for some character $\chi \in \mathrm{B}_{\pi}(G)$. In other words, $\alpha$ is a Fong character.

COROLlaRY 9.3. Continuing with the previous notation, assume that $\alpha$ and $\beta$ are Fong characters of $H$. Then $\alpha$ and $\beta$ belong to the same character $\chi \in \mathrm{B}_{\pi}(G)$ iff $\alpha^{G}=\beta^{G}$.

PROOF. The $\mathrm{B}_{\pi}$-characters to which $\alpha$ and $\beta$ belong are the unique irreducible constituents of $\alpha^{G}$ and $\beta^{G}$ that lie in $\mathrm{B}_{\pi}(G)$. In particular, if $\alpha^{G}=\beta^{G}$, then $\alpha$ and $\beta$ belong to the same character $\chi \in \mathrm{B}_{\pi}(G)$.

To prove the converse, it suffices to show that $\alpha^{G}$ can be computed from a knowledge of the character in $\mathrm{B}_{\pi}(G)$ to which $\alpha$ belongs. For $\psi \in \operatorname{Irr}(G)$, we need to be able to determine the multiplicity of $\psi$ in $\alpha^{G}$, and this, of course, is the multiplicity of $\alpha$ in $\psi_{H}$. By Corollary 9.1 , we can write

$$
\psi_{H}=\sum_{\chi \in \mathrm{B}_{\pi}(G)} a_{\chi} \chi_{H}
$$

and we see that the multiplicity of $\alpha$ in $\psi_{H}$ is exactly $a_{\chi}$, where $\alpha$ belongs to $\chi$.

There is an entirely different way to answer the two questions with which we began this section. Although computationally much less useful, this point of view actually gives additional insight into what is going on. 
Suppose $H \subseteq G$ and that $\alpha$ and $\beta$ are (possibly reducible) characters of $H$. We say that $\alpha$ and $\beta$ are linked in $G$ if we can find a subgroup $U \subseteq H$, a character $\gamma \in \operatorname{Char}(U)$ and an element $g \in G$ such that $U^{g} \subseteq H$ and such that $\gamma^{H}=\alpha$ and $\left(\gamma^{g}\right)^{H}=\beta$.

The relation 'linked' is clearly symmetric and reflexive, but it need not be transitive. We say, therefore, that characters $\alpha$ and $\beta$ of $H$ are connected in $G$ if there is a chain of links joining them.

What properties do two connected characters share? It is easy to see that linked characters (and hence connected characters) must have equal degrees. More generally, if $\alpha$ and $\beta$ are connected, then $\alpha^{G}=\beta^{G}$. There is one important property, however, that is not generally respected by character connectivity: irreducibility. Some connected components of characters of $H$ consist entirely of reducible characters, some components may contain both reducible and irreducible characters and some consist entirely of irreducible characters.

It may seem that the utility of the idea of character connectivity in $G$ would be severely limited by the fact that irreducible characters of $H$ may be connected to reducible characters. Actually, it is this aspect of connectivity which makes the notion useful.

THEOREM 9.4. Let $G$ be $\pi$-separable, let $H$ be a Hall $\pi$ subgroup of $G$ and suppose that $G$-connectivity of characters of $H$ is defined as in the foregoing discussion. Then the Fong characters of $H$ in $G$ are exactly those characters that lie in connected components consisting entirely of irreducible characters. Furthermore, two Fong characters of $H$ belong to the same character in $\mathrm{B}_{\pi}(G)$ iff they are $G$-connected.

Theorem 9.4 is the main result of [9], and we will not give the proof here. The following corollary, however, is easy to derive, and it has significance beyond the ambit of the $\pi$-theory we have been discussing.

COROLLARY 9.5. Let $H$ be a Hall $\pi$-subgroup of a $\pi$-separable group $G$ and suppose that $\alpha \in \operatorname{Ir}(H)$ is primitive. Then there exists $\chi \in \mathrm{B}_{\pi}(G)$ such that the Fong characters belonging to $\chi$ are exactly $\alpha$ and its conjugates under $\mathbf{N}_{G}(H)$.

PROOF. If $\alpha$ is $G$-linked to some character $\beta$ of $H$, then by definition we can find a subgroup $U \subseteq H$, a character $\gamma$ of $U$ and an element $g \in G$ such that $U^{g} \subseteq H$ and where $\gamma^{H}=\alpha$ and $\left(\gamma^{g}\right)^{H}=\beta$.

Since $\alpha$ is primitive, we must have $U=H, \gamma=\alpha$ and $g \in \mathbf{N}_{G}(H)$. It follows that $\beta=\alpha^{g}$ is conjugate to $\alpha$ in $\mathbf{N}_{G}(H)$, and in particular, $\beta$ is itself primitive. Thus the only characters that are $G$-connected to $\alpha$ are the conjugates of $\alpha$ under $\mathbf{N}_{G}(H)$, and it is easy to see that each of these is connected to (and in fact linked to) $\alpha$. The result now follows from Theorem 9.4. 
We close with an application to the theory of M-groups. If $G$ is an M-group, then it is solvable by Taketa's theorem, and thus for any set $\pi$ of primes, $G$ has a Hall $\pi$ subgroup $H$. It has been conjectured that Hall subgroups of M-groups are themselves necessarily M-groups, and we observe that if this were true, it would imply that the only primitive characters that $H$ could have would be linear. This consequence of the Hall subgroup conjecture is actually true, and this can be considered as (weak) evidence pointing toward the truth of the conjecture.

COROLLARY 9.6. Let $H$ be a Hall $\pi$-subgroup of an $M$-group $G$ and suppose that $\alpha \in \operatorname{Irr}(H)$ is primitive. Then $\alpha$ is linear.

PROOF. It follows from Corollary 9.5 that $\alpha$ is a Fong character belonging to some character $\chi \in \mathrm{B}_{\pi}(G)$. Since $G$ is an M-group, we know that $\chi$ is monomial, and thus by Corollary 6.6, some Fong character belonging to $\chi$ is monomial. By Corollary 9.5, however, we know that all of the Fong characters belonging to $\chi$ are conjugate in $\mathbf{N}_{G}(H)$, and hence all of them are monomial. Thus $\alpha$ is both primitive and monomial, and so it is linear.

\section{References}

[1] G. Amit and D. Chillag, 'On a question of Feit concerning character values of finite solvable groups', Pacific J. Math. 122 (1986), 257-261.

[2] P. Ferguson and A. Turull, 'On a question of Feit', Proc. Amer. Math. Soc. 97 (1986), 21-22.

[3] P. Fong, 'Solvable groups and modular representation theory', Trans. Amer. Math. Soc. 103 (1962), 484-494.

[4] D. Gajendragadkar, 'A characteristic class of characters of finite $\pi$-separable groups', J. Algebra 59 (1979), 237-259.

[5] I. M. Isaacs, Character theory of finite groups (Academic Press, New York, 1976).

[6] - 'Lifting Brauer characters of $p$-solvable groups II', J. Algebra 51 (1978), 476-490.

[7] _- 'Primitive characters, normal subgroups and M-groups', Math. Z. 177 (1981), 267-284.

[8] - 'Characters of $\pi$-separable groups', J. Algebra 86 (1984), 98-128.

[9] - 'Fong characters in $\pi$-separable groups', J. Algebra 99 (1986), 89-107.

[10] _ - 'Partial characters of $\pi$-separable groups', Prog. Math. 95 (1991), 273-287.

[11] G. Robinson and R. Staszewski, 'On the representation theory of $\pi$-separable groups', J. Algebra 119 (1988), 226-232.

[12] T. R. Wolf, 'Variations on McKay's character degree conjecture', J. Algebra 135 (1990), 123-138.

Mathematics Department

University of Wisconsin

Madison WI 53706

USA

e-mail: isaacs@math.wisc.edu 